\title{
Orofacial pain and temporomandibular disorders - the impact on oral health and quality of life ${ }^{*}$
}

\section{Paulo César Rodrigues Conti(a) Lívia Maria Sales Pinto-Fiamengui(a) Carolina Ortigosa Cunha(a) Ana Cláudia de Castro Ferreira Conti(b)}
(a) Department of Prosthodontics, Bauru School of Dentistry, Univ de São Paulo - USP, Bauru, SP, Brazil.

(b) Department of Dentistry, Univ Norte do Paraná - UNOPAR, Londrina, PR, Brazil.

\begin{abstract}
Many conditions may cause painful symptoms in orofacial structures. Among the chronic conditions that affect this area, temporomandibular disorders are the most common. Temporomandibular Disorder is a collective term that includes a number of clinical complaints involving the masticatory muscles, the Temporomandibular Joint and associated structures. In some cases, these complaints can be associated with depression, catastrophizing behavior and impact on quality of life. The present study aims to explain the relationship between Temporomandibular Disorders and pain chronification and their relation to a variety of psychosocial and behavioral comorbid conditions. The mechanisms of pain conduction and suggestions for management are also addressed.
\end{abstract}

Descriptors: Temporomandibular Joint Disorders; Facial Pain; Chronic Pain; Quality of Life.

\section{Introduction Orofacial pain and quality of life}

Orofacial Pain is a term that comprises different manifestations of pain in the face and oral cavity. It is a broad classification, including many conditions highly prevalent in the general population, such as pulpal and periodontal processes, sinusitis, trigeminal neuralgia, and masticatory muscle and temporomandibular joint (TMJ) pain. The latter two conditions fall under a group of conditions named temporomandibular disorders (TMDs). TMD is a collective term embracing a number of clinical problems that involve the masticatory muscles and/or temporomandibular joints (TMJ) and associated structures. ${ }^{1}$ It is recognized as the most common non-odontogenic-related chronic orofacial pain condition confronted by dentists and other healthcare providers. ${ }^{2}$ The most frequent symptom is pain, usually located in the masticatory muscles, pre-auricular area, and/or TMJ. In addition to pain, which is usually aggravated by chewing and other jaw functions, patients with TMD often have limited or asymmetric mandibular movements and joint sounds, commonly described as clicking, popping, grating, or crepitus. ${ }^{3}$ TMD patients also describe symptoms of pain and dysfunction affecting ears, eyes and/or throat, as well as headaches, which involve some or all of the frontal, temporal, parietal, occipital and neck regions. ${ }^{4}$

In cases of persistent and recurrent pain, TMD may follow a chronic course. In these cases, although TMD is not a life-threatening disease, the
Submitted: Sep 09, 2012

Last revision: Oct 23, 2012
Accepted for publication: Oct 10, 2012

\section{Corresponding Author:}

E-mail: pcconti@fob.usp.br 
patients' quality of life may be reduced. ${ }^{5}$ Because of this presumed reduction in quality of life in TMD patients, TMDs are now studied and treated from a medical perspective that involves a biopsychosocial understanding of how chronic pain disorders affect those who have them. ${ }^{6}$

According to Barros et al., orofacial pain has a great impact on the quality of life of individuals with TMD, with no difference between genders. However, there is a clear correlation between the severity of TMD and the impact on the quality of life of individuals with TMD seeking treatment. ${ }^{7}$

The present critical review of the literature aims to explain the impact of orofacial pain conditions, including that of TMD, on oral health and quality of life.

\section{Discussion}

Pain appears to be a key symptom associated with possible impairment of oral-health-related quality of life (OHQoL) ${ }^{8}$ It is well know and widely accepted that, before being perceived by the patient as pain in the Central Nervous System (CNS), the initial nociceptive stimulus is modulated by different descending mechanisms. The imbalance between the amount of stimuli and the efficacy of modulation mechanisms is processed as a pain sensation. It is also well-documented that either high magnitude or repetitive nociceptive impulses cause peripheral and central neuronal changes, leading to the maintenance and exacerbation of the pain sensation. These alterations are known as "sensitization," often irreversible, and responsible for patients' reporting long-term pain, even after many unsuccessful treatments. Based on this, it is thought that chronic orofacial pain not only occurs due to peripheral input, but also to CNS changes. ${ }^{9}$

In addition to all the biological deficits caused by the maintenance of a pain sensation and imbalance in the modulation system, as described above, chronic patients are frequently affected by a variety of psychosocial and behavioral comorbid conditions, such as impaired quality of life, depression and sleep disorders, among others. The fact is that all the circuits involved in depression and sleep problems are very similar to those present in chronic pain, and must be contemplated in the treatment of patients experienc- ing orofacial pain.

In addition to biomechanical factors (e.g. dental occlusion and bruxism), ${ }^{10,11}$ psychological factors, like stress ${ }^{11}$ and depression, ${ }^{12}$ have also been related to TMD, especially chronic TMD conditions. It is well-reported that a considerable number of TMD patients are clinically depressed, and this depression may influence their response to conventional therapies. $^{2}$

Patients with diverse chronic pain problems, including that of TMD, present some reactions, such as pain-related beliefs, catastrophizing (expecting or worrying about major negative consequences from a situation, even one of minor importance), and coping strategies (e.g. coping self-statements). Pain-related catastrophizing may be defined as an excessive focus on pain, magnification of the threat associated with pain, and a feeling of helplessness in controlling pain. Pain-related catastrophizing may represent another potentially powerful non-biological factor that influences symptoms and functioning among TMD patients. ${ }^{13}$ These reactions are strongly associated with pain intensity, psychosocial adjustment, and physical functioning, ${ }^{14}$ as well as impairment of quality of life.

Today, the most popular theories regarding TMD etiology are based on the biopsychosocial model, which involves a combination of biological, psychological and social factors that provide an excellent descriptor of the world that most patients with pain (especially patients with chronic conditions) are living in. They have a biological problem that may have psychological antecedents as well as behavioral consequences. This situation exists in a social framework that includes interpersonal relationships with friends, families and health care providers, who almost always produce major negative experiences for the patients as well as for their immediate families. Unlike the mechanistic dental theories of etiology, the biopsychosocial model encourages a rehabilitation-management approach rather than providing the unrealistic expectation of a permanent cure (which is even less likely in chronic conditions). ${ }^{15}$

Recognition and treatment of TMD is over a century old, and wide-ranging treatment philosophies have evolved over this time. ${ }^{16}$ The treatment 
of TMDs by conservative and reversible approaches has proved appropriate and successful. According to Barros et al., subjective indicators of oral health should confirm the success of TMD treatment, reducing its signs and/or symptoms and reestablishing the orofacial function. ${ }^{7}$

The treatment of acute TMD is based on an occlusal splint (Figure 1), pharmacotherapy, and physical therapy (Figure 2), among others. Behavioral changes, such as the reduction of stress levels and adequate, uninterrupted sleep, are also very important in the management of patients suffering from TMD. Sleep is necessary for joints and muscles to undergo a period of physiologic rest, recovery, and repair. ${ }^{16}$

Psychological therapies established for other chronic pain conditions may be useful in lending support to patients who are managing persistent pain and social debilitation, and enduring stress in a life associated with TMD and other forms of orofacial pain. ${ }^{2}$

Studies of patients with TMD have shown that many of them, especially females, experience a multitude of other functional (nonorganic) disorders, such as fibromyalgia, interstitial cystitis, irritable bowel syndrome and pelvic pain, while others have reported multiple pain sites throughout their bodies. ${ }^{17}$

These high levels of comorbidity with other conditions have given rise to hypotheses about centrally mediated deregulatory problems producing multiple symptoms in susceptible patients. ${ }^{15}$ In an effort to better understand and treat these disorders, they were unified under one term called "central sensitiv-

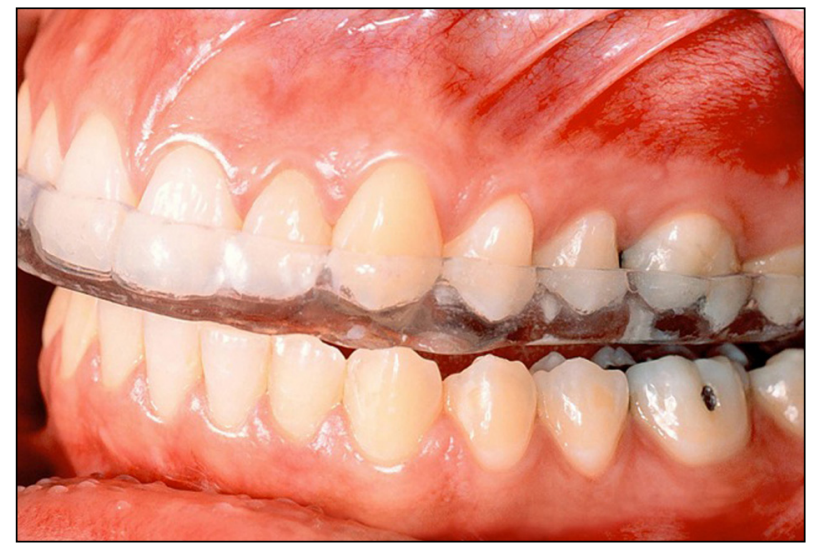

Figure 1 - Occlusal splint used as treatment for TMD. ity syndromes," also called "functional syndromes," "functional somatic syndromes," or "somatoform syndromes". Given the psychosomatic lens through which many of these disorders are viewed, demonstration of evidence-based links supporting shared pathophysiology between these disorders could provide direction to clinicians and researchers working to treat these diagnoses. ${ }^{18}$

As explained above, although the onset of several of these disorders is triggered by peripheral painproducing mechanisms, persistent nociceptive inputs lead to changes in the central nociceptive system. ${ }^{18}$ Central sensitization is common to these central sensitivity syndromes, and is clinically and physiologically characterized by hyperalgesia (excessive sensitivity to a normally painful stimulus), allodynia (painful sensation to a normally nonpainful stimulus), expansion of the receptive field (pain beyond the area of peripheral nerve supply), prolonged electrophysiological discharge, and unpleasant afterstimulus quality of the pain (e.g., burning, throbbing, tingling or numbness). ${ }^{19}$ The rehabilitation of patients with chronic musculoskeletal pain like that of chronic TMDs should target, or at least take into account, the process of central sensitization..$^{20}$

Dahlström and Carlsson performed a systematic review of TMD and oral-health-related quality of life (OHRQoL) and concluded that subjective TMD symptoms had a greater impact than clinical findings, and the more painful and severe the TMDs, the greater the impact on OHRQoL. In their systemat-

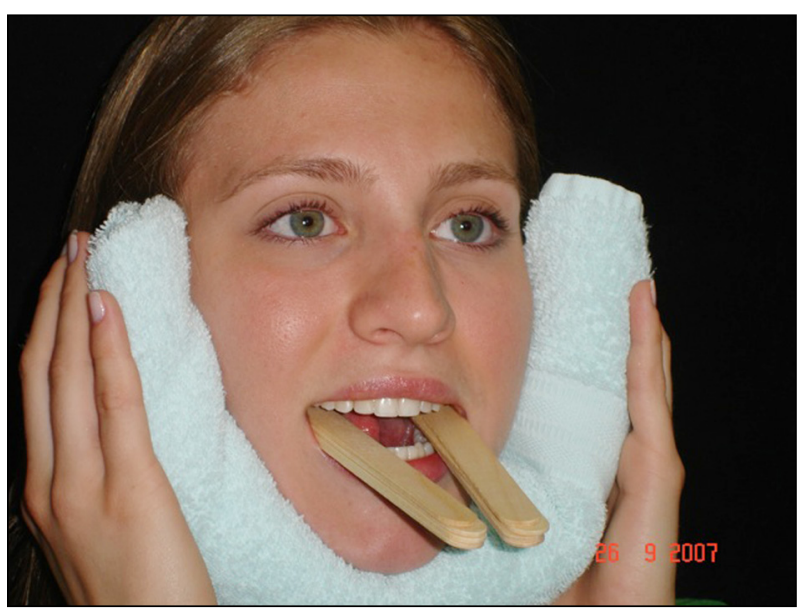

Figure 2 - Physical therapy used as treatment for TMD. 
ic review, some, but not all, reports suggested that TMDs have a greater impact on OHQoL than other orofacial diseases/illnesses or conditions. ${ }^{21}$

\section{Conclusion}

In order to understand and manage TMD patients, it is necessary to understand temporomandibular disorders and their comorbidities from a medical perspective, and try to observe how quality of life is reduced, on an individual basis, patient-to-

\section{References}

1. McNeill C, Mohl ND, Rugh JD, Tanaka TT. Temporomandibular disorders: diagnosis, management, education, and research. J Am Dent Assoc. 1990 Mar;120(3):253-7.

2. McNeill C. Management of temporomandibular disorders: concepts and controversies. J Prosthet Dent. 1997 May;77(5):510-22.

3. Yap AU, Tan KB, Chua EK, Tan HH. Depression and somatization in patients with temporomandibular disorders. J Prosthet Dent. 2002 Nov;88(5):479-84.

4. Suvinen TI, Reade PC, Kemppainen P, Könönen M, Dworkin SF. Review of aetiological concepts of temporomandibular pain disorders: towards a biopsychosocial model for integration of physical disorder factors with psychological and psychosocial illness impact factors. Eur J Pain. 2005 Dec;9(6):613-33.

5. Liu HX, Liang QJ, Xiao P, Jiao HX, Gao Y, Ahmetjiang A. The effectiveness of cognitive-behavioural therapy for temporomandibular disorders: a systematic review. J Oral Rehabil. 2012 Jan;39(1):55-62.

6. Dworkin SF, Massoth DL. Temporomandibular disorders and chronic pain: disease or illness? J Prosthet Dent. 1994 Jul;72(1):29-38.

7. Barros VM, Seraidarian PI, Cortes MI, Paula LV. The impact of orofacial pain on the quality of life of patients with temporomandibular disorder. J Orofac Pain. 2009 Winter;23(1):28-37.

8. Türp JC, Motschall E, Schindler HJ, Heydecke G. In patients with temporomandibular disorders, do particular interventions influence oral health-related quality of life? A qualitative systematic review of the literature. Clin Oral Implants Res. 2007 Jun;18 Suppl 3:127-37.

9. Conti PC, Pertes RA, Heir GM, Nasri C, Cohen HV, Araújo CR. Orofacial pain: basic mechanisms and implication for successful management. J Appl Oral Sci. 2003 Mar;11(1):1-7.

10. Marklund S, Wänman A. Risk factors associated with incidence and persistence of signs and symptoms of temporomandibular disorders. Acta Odontol Scand. 2010 Sep;68(5):289-99. patient, considerations that must be borne in mind in the management of the patient. For the dental profession, the implications of this information are serious, especially regarding chronic TMD conditions, in which modulation mechanisms of pain are reduced and comorbidities are present most of the time. The use of biopsychosocial management strategies to reduce pain and improve function is the approach that has proved successful for most TMD and orofacial pain patients.

11. Akhter R, Morita M, Esaki M, Nakamura K, Kanehira T. Development of temporomandibular disorder symptoms: a 3-year cohort study of university students. J Oral Rehabil. 2011 Jun;38(6):395-403.

12. Sullivan MJ, Thorn B, Haythornthwaite JA, Keefe F, Martin M, Bradley LA, et al. Theoretical perspectives on the relation between catastrophizing and pain. Clin J Pain. 2001 Mar;17(1):52-64.

13. Klasser GD, Greene CS. The changing field of temporomandibular disorders: what dentists need to know. J Can Dent Assoc. 2009 Feb;75(1):49-53.

14. Dym H, Israel H. Diagnosis and treatment of temporomandibular disorders. Dent Clin North Am. 2012 Jan;56(1):149-61.

15. Liao CH, Chang CS, Chang SN, Lane HY, Lyu SY, Morisky DE, et al. The risk of temporomandibular disorder in patients with depression: a population-based cohort study. Community Dent Oral Epidemiol. 2011 Dec;39(6):525-31.

16. Geisser ME, Robinson ME, Riley JL. Pain beliefs, coping, and adjustment to chronic pain: let's focus more on the negative. Pain Forum. 1999 Winter;8(4):161-68.

17. de Leeuw R, Klasser GD, Albuquerque RJ. Are female patients with orofacial pain medically compromised?. J Am Dent Assoc. 2005 Apr;136(4):459-68.

18. Kindler LL, Bennett RM, Jones KD. Central sensitivity syndromes: mounting pathophysiologic evidence to link fibromyalgia with other common chronic pain disorders. Pain Manag Nurs. 2011 Mar;12(1):15-24.

19. Yunus MB. Central sensitivity syndromes: a new paradigm and group nosology for fibromyalgia and overlapping conditions, and the related issue of disease versus illness. Semin Arthritis Rheum. 2008 Jun;37(6):339-52.

20. Nijs J, Paul Van Wilgen C, Van Oosterwijck J, Van Ittersum M, Meeus M. How to explain central sensitization to patients with 'unexplained' chronic musculoskeletal pain: practice guidelines. Man Ther. 2011 Oct;16(5):413-8.

21. Dahlström L, Carlsson GE. Temporomandibular disorders and oral health-related quality of life. A systematic review. Acta Odontol Scand. 2010 Mar;68(2):80-5. 